\title{
MENSURAÇÃO DA GRAVIDADE DE QUADROS CLÍNICOS RESULTANTES DE CIRURGIAS: ESTIMAÇÃO DE MAGNITUDES VERSUS ESTIMAÇÃO DE CATEGORIAS
}

\author{
Fátima Aparecida Emm Faleiros Sousa* \\ Patrícia Bodnar Giuntini**
}

\begin{abstract}
SOUSA, F.A.E.F; GIUNTINI, P.B. Mensuração da gravidade de quadros clínicos resultantes de cirurgias: estimação de magnitudes versus estimação de categorias. Rev.latino-am.enfermagem, Ribeirão Preto, v. 9, n. 1, p. 96-101, janeiro 2001.
\end{abstract}

A gravidade de quadros clínicos resultantes de cirurgias foi escalonada pelos métodos psicofísicos de estimação de magnitudes e de categorias por 20 médicos e 20 enfermeiros. Os resultados mostraram: 1) o contínuo de gravidade de quadros clínicos resultantes de cirurgias tem caracteristicas quantitativas ou protéticas, e não qualitativas ou metatéticas; 2) a variabilidade das estimativas de magnitudes é uma função linear da magnitude das estimativas, segundo a lei de Ekman.

UNITERMOS: gravidade, psicofisica

\section{INTRODUÇÃO}

$\boldsymbol{O}$ método de estimação de magnitudes foi utilizado com sucesso na mensuração da gravidade de diferentes enfermidades. De fato, WYLER et al. (1968) elaboraram uma lista contendo 126 enfermidades a qual foi enviada pelo correio a duas amostras distintas: uma não médica e a outra médica. A tarefa dos sujeitos consistia em estimar a magnitude da gravidade das enfermidades assinalando a cada uma delas um número que fosse proporcional ao valor de 500 designado à enfermidade da úlcera péptica. Exemplos de algumas enfermidades foram: constipação intestinal, enxaqueca, diarréia, sinusite, acne, astigmatismo, menopausa, menstruação, eczema, alergia medicamentosa, gonorréia, coma, depressão, epilepsia, derrame cerebral, ataque cardíaco, uremia, câncer e leucemia. As duas amostras foram altamente concordantes em suas estimativas de magnitudes de cada uma dessas enfermidades bem como em suas respectivas ordenações. Os resultados também indicaram que as variáveis idade, sexo, estado civil, etc., afetam numa extensão maior os julgamentos feitos pela amostra não médica do que aqueles feitos pela amostra médica. Combinando as estimativas de magnitudes de ambas as amostras, estas indicaram que a caspa foi a enfermidade com menor estimativa de magnitude, o aborto foi uma enfermidade com estimativa de magnitude mediana e a leucemia foi a enfermidade com maior estimativa de magnitude. O mesmo padrão de resultados foi obtido por WYLER et al. (1971) e VOLICER \& BOHANNON (1975).

Em outro estudo similar, WYLER et al. (1970) replicaram o trabalho original usando uma outra amostra de médicos e analisando as estimativas em função de suas respectivas especialidades médicas. Os resultados mostraram que as estimativas de magnitudes numéricas feitas por médicos de diferentes especialidades não foram significativamente diferentes entre si, exceto apenas para 5 enfermidades, indicando, portanto, que a variável especialidade do respondente não é significativa.

No estudo de FALEIROS SOUSA et al. (1998) o objetivo foi escalonar a gravidade de 68 quadros clínicos cirúrgicos por médicos e enfermeiros através do método de estimação de magnitudes. Os dados permitiram concluir que os quadros clínicos-cirúrgicos aneurismectomia de aorta, aneurismectomia cerebral e revascularização do miocárdio foram considerados os de maior gravidade, enquanto que os quadros clínicoscirúrgicos adenoidectomia, amigdalectomia e curetagem uterina os de menor gravidade em todas as escalas obtidas pelos diferentes métodos psicofísicos diretos; o contínuo não métrico de gravidade de quadros clínicos-cirúrgicos possui características protéticas; a relação entre as estimativas do emparelhamento de forças dinamométricas e as estimativas do emparelhamento de

\footnotetext{
* Professor Associado junto ao Departamento de Enfermagem Geral e Especializada da Escola de Enfermagem de Ribeirão Preto da Universidade de São Paulo. Endereço: Av. Bandeirantes, 3900 - Monte Alegre - 14040-902 - Ribeirão Preto - São Paulo - Brasil ** Aluna da Pós-Graduação da Escola de Enfermagem de Ribeirão Preto da Universidade de São Paulo
} 
comprimentos de linhas é uma função de potência com um expoente não significativamente diferente de $0,77 \mathrm{e}$ finalmente a escala de razão de gravidade de quadros clínicos-cirúrgicos é válida, estável e consistente, pois o expoente obtido através dos emparelhamentos de comprimentos de linhas e de forças dinamométricas não foi significativamente diferente do expoente predito pela propriedade de transitividade das escalas, ou seja, daquele obtido nas tarefas de calibração (Para detalhes sobre a metodologia ver FALEIROS SOUSA \& DA SILVA (1996a,b); GESCHEIDER (1997); PEREIRA \& FALEIROS SOUSA (1998a,b); FALEIROS SOUSA et al. (1998).

Deste estudo foram selecionados 15 quadros clínicos-cirúrgicos considerando-se aqueles que apresentavam uma determinada diferença (espaçamento) entre as estimativas de magnitudes para facilitar a discriminação dos quadros clínicos nos julgamentos feitos pelos participantes, ou seja, não foram escolhidos quadros clínicos resultantes com estimativas de magnitudes adjacentes muito próximas.

Os objetivos desse experimento foram: 1) Comparar as escalas derivadas de julgamentos intervalares (estimativas de categorias) com as escalas derivadas de julgamentos de razão (estimativas de magnitudes); 2) Verificar através da comparação entre as estimativas de magnitudes e as estimativas de categorias se o contínuo não métrico de gravidade de quadros clínicos resultantes de cirurgias tem características protéticas ou metatéticas; 3) Verificar se as ordenações dos graus de gravidade de quadros clínicos resultantes de cirurgias derivadas dos dois métodos psicofísicos são similares entre si; 4) Verificar a estabilidade e/ou equivalência das escalas de categorias e de razão através de duas modalidades de respostas diferentes, quais sejam numérica sem limites (estimativas de magnitudes) e numérica com limites (estimativas de categorias) e 5) Verificar a estabilidade e/ou equivalência dessas escalas entre as duas amostras.

Esse estudo justifica-se pelo fato de que a estratégia psicofísica é superior às inúmeras outras estratégias comumente utilizadas em Enfermagem por fornecer informações quantitativas sobre a intensidade dos julgamentos dos pacientes, médicos e enfermeiros (SENNOTT-MILLER, et al., 1988; MEEK et al., 1992.

\section{MÉTODO}

\subsection{Participantes}

Participaram 40 sujeitos, sendo 20 médicos (amostra A1) e 20 enfermeiros (amostra A2), profissionais atuantes na cidade de Ribeirão Preto, com idades variando entre 22 e 45 anos e todos eram ingênuos, ou seja, os sujeitos não tinham experiência prévia com experimentos dessa natureza.

\subsection{Material}

Foram elaborados quatro blocos de papel contendo na primeira página instruções específicas para cada tipo de método psicofísico e na página seguinte uma lista de 15 quadros clínicos resultantes de cirurgias selecionados do estudo de FALEIROS SOUSA et al. (1998).

\subsection{Procedimento}

Os métodos psicofísicos utilizados foram estimação de magnitudes e estimação de categorias. A tarefa dos participantes no primeiro método foi assinalar um número a cada cirurgia que fosse proporcional a quantidade de gravidade do quadro clínico resultante. Dessa forma, se o participante julgasse que um dado quadro clínico resultante de uma dada cirurgia possuísse duas vezes mais gravidade do que um outro quadro clínico, ele deveria assinalar a ele um número duas vezes maior. Se ele julgasse que um dado quadro clínico resultante de uma dada cirurgia possuísse metade da gravidade do que um outro quadro clínico, deveria assinalar a ele um número que fosse metade do atribuído aquele quadro clínico. As diferentes cirurgias foram apresentadas em um série de 15, as quais foram dispostas em cada página contendo em média vinte cirurgias, numa ordem totalmente aleatória para todos participantes. Cada participante estabeleceu 15 estimativas, sendo uma para cada cirurgia. Estímulo padrão e módulo foram previamente, tendo sido estabelecido o valor de 100 para COLECISTECTOMIA.

No segundo método, a tarefa dos participantes foi assinalar um escore, o qual variava de 1 a 7 , a cada cirurgia em função do grau de severidade dos quadros clínicos resultantes. O participante foi instruído a assinalar à cirurgia cujo quadro clínico fosse considerado o de maior gravidade o escore máximo de 7 e, ao de menor gravidade, o escore mínimo de 1 . Os outros escores intermediários, de 2 a 6 , deveriam ser utilizados para indicar graus intermediários de severidade que os quadros clínicos resultantes possuem. Previamente não foram estabelecidos os quadros clínicos resultantes de cirurgias de graus de severidades máximo e mínimo. As diferentes cirurgias foram apresentadas em uma série de 15 , as quais foram dispostas em uma página, numa ordem totalmente aleatória para todos participantes. Cada participante estabeleceu 15 estimativas, sendo uma para cada cirurgia.

As instruções dadas aos participantes, independente do método psicofísico utilizado, requeriam que os julgamentos fossem realizados em função do grau 
relativo da gravidade dos quadros clínicos resultantes de cirurgias, considerando a maioria dos pacientes, cujo problema central fosse este quadro clínico e as outras condições relativamente controladas. Os mesmos 40 participantes fizeram as duas tarefas, sendo que metade julgou primeiro pelo método de estimação de magnitudes e depois pelo método de estimação de categorias, e a outra metade julgou na ordem inversa. Para verificar se a relação entre escalas de magnitudes e escalas de categorias é independente do tipo de quadro clínicocirúrgico, utilizamos 15 cirurgias para os médicos e outras 15 , sendo algumas diferentes para os enfermeiros (Tabelas 1 e 2). O experimento foi realizado ora em laboratório, ora em residências, ora em locais de trabalho do participante, sendo que o instrumento era entregue e posteriormente recolhido, após os participantes serem instruídos a respondê-lo individualmente e sem interrupção. Os sujeitos foram orientados a colaborar se quisessem para a pesquisa, sendo que não houve recusa nas participações após serem lidas as instruções.

\section{RESULTADOS E DISCUSSÃO}

As estimativas médias para os diferentes quadros clínicos realizadas pelos dois grupos de participantes e as respectivas ordenações de posições deste estudo de FALEIROS SOUSA et al. (1998), estão apresentadas nas Tabelas 1 e 2, respectivamente, para as amostras A1 e A2, para os métodos psicofísicos utilizados: Estimação de categorias (EC) e Estimação de magnitudes (EM).

Tabela 1 - Média geométrica das estimativas de magnitudes (EM), média aritmética das estimativas de categorias (EC) e ordenação das posições (OP) de gravidade julgada de cada quadro clínico considerando a amostra $A 1$

\begin{tabular}{l|c|c|c|c}
\hline \multicolumn{1}{c|}{ CIRUR GIAS } & EM & OP & EC & OP \\
\hline Adenoidectomia & 38,71 & $4^{\circ}$ & 1,7 & $3^{\circ}$ \\
\hline Aneurismectomia cerebral & 374,30 & $12^{\circ}$ & 5,4 & $13^{\circ}$ \\
\hline Artrodese de coluna cervical & 168,10 & $10^{\circ}$ & 3,7 & $11^{\circ}$ \\
\hline Gastrostomia & 38,42 & $3^{\circ}$ & 2 & $5^{\circ}$ \\
\hline Arrigdalectomia & 30,28 & $2^{\circ}$ & 1,2 & $2^{\circ}$ \\
\hline Fechamento de ileostomia & 41,29 & $5^{\circ}$ & 1,8 & $4^{\circ}$ \\
\hline Esofagectomia & 275,85 & $13^{\circ}$ & 4,5 & $12^{\circ}$ \\
\hline Colecistectomia & 100,00 & $8^{\circ}$ & 2,4 & $6^{\circ}$ \\
\hline Simpatectomia & 80,89 & $6^{\circ}$ & 2,5 & $7^{\circ}$ \\
\hline Fechamento de cistostomia & 26,64 & $1^{\circ}$ & 1,1 & $1^{\circ}$ \\
\hline Retossigmoidec tornia & 173,21 & $11^{\circ}$ & 3,4 & $9^{\circ}$ \\
\hline Aneurismectomia de aorta & 378,75 & $14^{\circ}$ & 5,8 & $15^{\circ}$ \\
\hline Mastectomiatipo Patey & 135,97 & $9^{\circ}$ & 3,5 & $10^{\circ}$ \\
\hline Revascularizaçäo do miocárdio & 385,24 & $15^{\circ}$ & 5,5 & $14^{\circ}$ \\
\hline Aritenoidectomia & 89,37 & $7^{\circ}$ & 2,6 & $8^{\circ}$ \\
\hline
\end{tabular}

Tabela 2 - Média geométrica das estimativas de magnitudes (EM), média aritmética das estimativas de categorias (EC) e ordenação das posições (OP) de gravidade julgada de cada quadro clínico considerando a amostra $\mathrm{A2}$

\begin{tabular}{l|c|c|c|c}
\hline \multicolumn{1}{c|}{ CIRUR GIAS } & EM & OP & EC & OP \\
\hline Amigdalectomia & 51,83 & $3^{\circ}$ & 1,5 & $1^{\circ}$ \\
\hline Revascularizaçäo do miocárdio & 279,60 & $15^{\circ}$ & 5,6 & $15^{\circ}$ \\
\hline Gastrostomia & 62,70 & $4^{\circ}$ & 2,8 & $6^{\circ}$ \\
\hline Matectomiatipo Patey & 154,10 & $12^{\circ}$ & 4,5 & $12^{\circ}$ \\
\hline Colecistectomia & 100,00 & $9^{\circ}$ & 3,6 & $8^{\circ}$ \\
\hline Vesicostomia & 85,44 & $6^{\circ}$ & 2,6 & $4^{\circ}$ \\
\hline Laparotomia Exploradora & 80,99 & $5^{\circ}$ & 3,8 & $9^{\circ}$ \\
\hline Histerectomia vaginal & 95,02 & $7^{\circ}$ & 3,5 & $7^{\circ}$ \\
\hline Dacriocistominostomia & 46,27 & $2^{\circ}$ & 1,5 & $2^{\circ}$ \\
\hline Gastrectomia total & 195,60 & $13^{\circ}$ & 4,5 & $13^{\circ}$ \\
\hline Aneurismectomia de aorta & 277,30 & $14^{\circ}$ & 5,2 & $14^{\circ}$ \\
\hline Antrectomia & 98,95 & $8^{\circ}$ & 2,7 & $5^{\circ}$ \\
\hline Fechamento de cistostomia & 38,42 & $1^{\circ}$ & 2,2 & $3^{\circ}$ \\
\hline Trombectomia & 148,10 & $11^{\circ}$ & 3,9 & $10^{\circ}$ \\
\hline Vulvectomia & 118,95 & $10^{\circ}$ & 4,2 & $11^{\circ}$ \\
\hline
\end{tabular}

Pode-se observar nestas Tabelas, que independente do método psicofísico utilizado, os quadros clínicos resultantes de fechamento de cistostomia, amigdalectomia e adenoidectomia foram considerados os de menor gravidade para os médicos e amigdalectomia, fechamento de cistostomia e dacriocistorrinostomia para os enfermeiros, enquanto que os quadros clínicos resultantes aneurismectomia de aorta, revascularização do miocárdio e aneurismectomia cerebral os de maior gravidade para os médicos e revascularização do miocárdio, aneurismectomia de aorta e gastrectomia total para os enfermeiros. De fato, o coeficiente de concordância de Kendall (W) mostrou que as diferentes amostras foram altamente concordantes quanto à ordenação desses diferentes quadros clínicos. Esses valores do coeficiente de concordância foram $\mathrm{W}=0,92$ para o método de estimação de categorias, 0,97 para o de estimação de magnitude, sendo ambos altamente significativos $(\mathrm{p}<0,001)$.

Um coeficiente de concordância aplicado às estimativas efetuadas por meio de cada método, para os 15 quadros clínicos mostrou $\mathrm{W}=0,98$ para os médicos e 0,96 para os enfermeiros, sendo ambos altamente significativos $(\mathrm{p}<0,001)$, indicando, portanto, que a ordenação por postos derivada das estimativas de cada método é altamente concordante para as duas amostras. Por exemplo, o quadro clínico resultante de aneurismectomia cerebral ocupa a $13^{a}$ posição e o quadro clínico resultante de amigdalectomia ocupa a $2^{\mathrm{a}}$ posição nos dois tipos de contínuos de respostas da 
amostra de médicos. Da mesma forma, o quadro clínico resultante de histerectomia vaginal ocupa a $7^{\mathrm{a}}$ posição e o quadro clínico resultante de revascularizaçãa do miocárdio ocupa a $15^{\mathrm{a}}$ posição nos dois tipos de contínuos de respostas da amostra de enfermeiros.

Para verificar se o contínuo de gravidade de quadros clínicos resultantes de cirurgias tem características protéticas ou metatéticas, como ocorre com os contínuos sensoriais, as médias aritméticas das estimativas de categorias foram projetadas em função das médias geométricas das estimativas de magnitudes dos quadros clínicos resultantes. Em coordenadas lineares a relação entre essas estimativas mostrou uma leve concavidade descendente. Também, quando as médias aritméticas das estimativas de categorias foram projetadas em função dos logaritmos das médias geométricas das estimativas de magnitudes, a relação mostrou uma concavidade ascendente. As Figuras 1 e 2 mostram claramente esta relação em coordenadas lineares e as Figuras 3 e 4 em coordenadas mono-logarítmicas. Tomadas juntas, fica demonstrado que o contínuo de gravidade de quadros clínicos resultantes de cirurgias tem características de contínuo protético ou aditivo, independente do grupo de cirurgias julgado.

Outro resultado interessante é a relação entre os desvios padrão da média geométrica e as médias geométricas das estimativas de magnitudes (Figuras $5 \mathrm{e}$ 6). Observa-se que a relação é linear, de modo que quanto maior a estimativa de magnitude, tanto maior é o desvio padrão da média. Portanto, este padrão de resultados mostra o princípio geral conhecido como Lei de Ekman (STEVENS, 1966, 1975), de que a variabilidade das estimativas é uma função linear da magnitude das estimativas. De outro lado, a mesma relação não ocorre com as estimativas de categorias, uma vez que nas categorias extremas, geralmente o desvio padrão aritmético é pequeno, e no restante, usualmente é constante ou aumenta em função da categoria média (Figuras 7 e 8). Este mesmo padrão de resultados ocorre para os diferentes grupos de cirurgias.

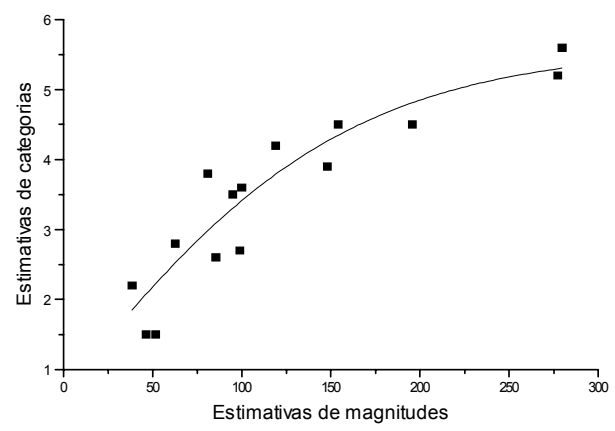

Figura 1 - Relação entre as médias aritméticas das estimativas de categorias e as médias geométricas das estimativas de magnitudes da gravidade de quadros clínicos resultantes de cirurgias em coordenadas lineares. Dados da Amostra 1

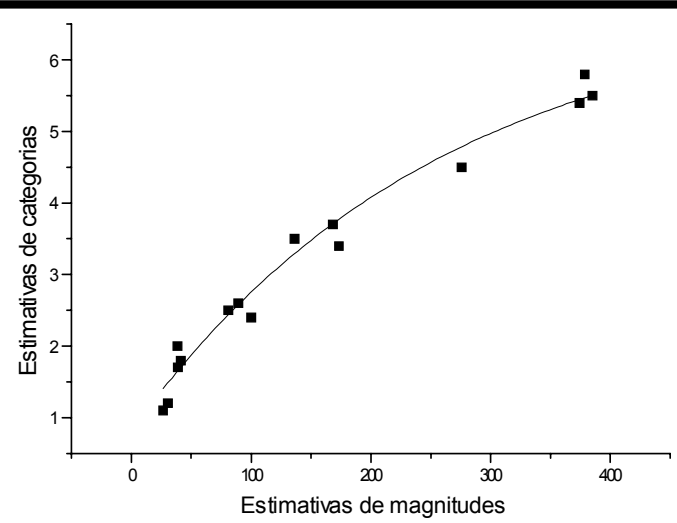

Figura 2 - Relação entre as médias aritméticas das estimativas de categorias e as médias geométricas das estimativas de magnitudes da gravidade de quadros clínicos resultantes de cirurgias em coordenadas lineares. Dados da Amostra 2

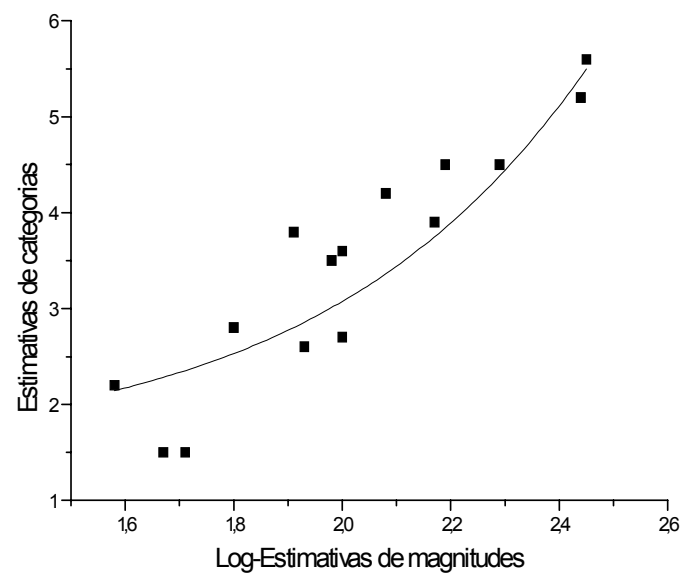

Figura 3 - Relação entre as médias aritméticas das estimativas de categorias e os logaritmos das médias geométricas das estimativas de magnitudes da gravidade de quadros clínicos resultantes de cirurgias. Dados da Amostra 1

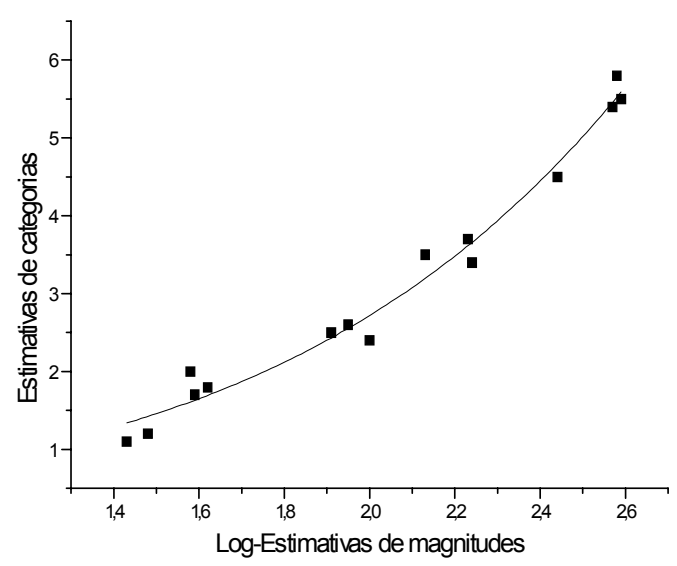

Figura 4 - Relação entre as médias aritméticas das estimativas de categorias e os logaritmos das médias geométricas das estimativas de magnitudes da gravidade de quadros clínicos resultantes de cirurgias. Dados da Amostra 2 


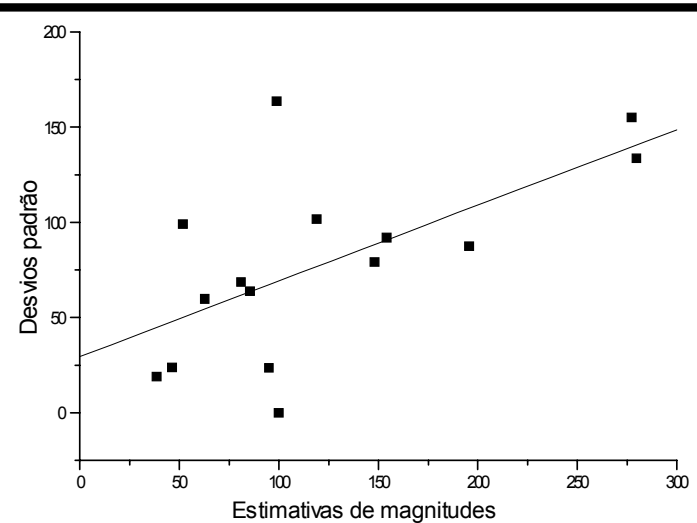

Figura 5 - Desvio padrão da média geométrica em função da média geométrica das estimativas de magnitudes da gravidade de quadros-clínicos resultantes de cirurgias. Dados da Amostra 1

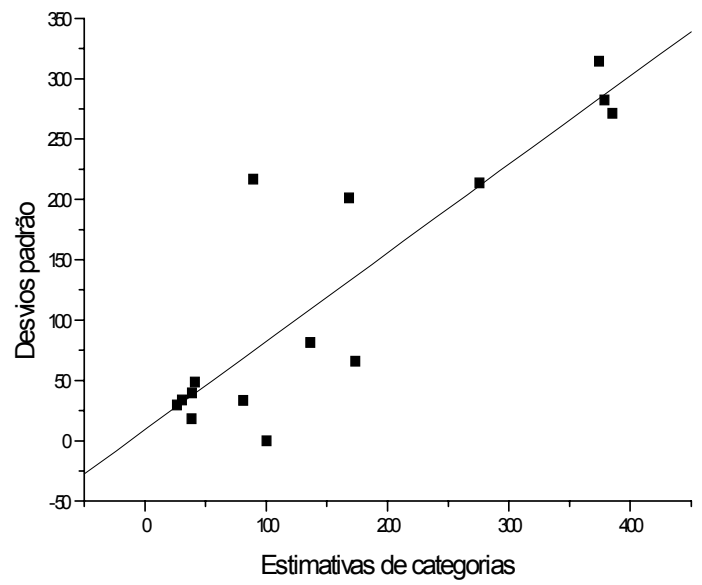

Figura 6 - Desvio padrão da média geométrica em função da média geométrica das estimativas de magnitudes da gravidade de quadros clínicos resultantes de cirurgias. Dados da Amostra 2



Figura 7 - Desvio padrão da média aritmética em função da média aritmética das estimativas de categorias da gravidade de quadros clínicos resultantes de cirurgias. Dados da Amostra 1

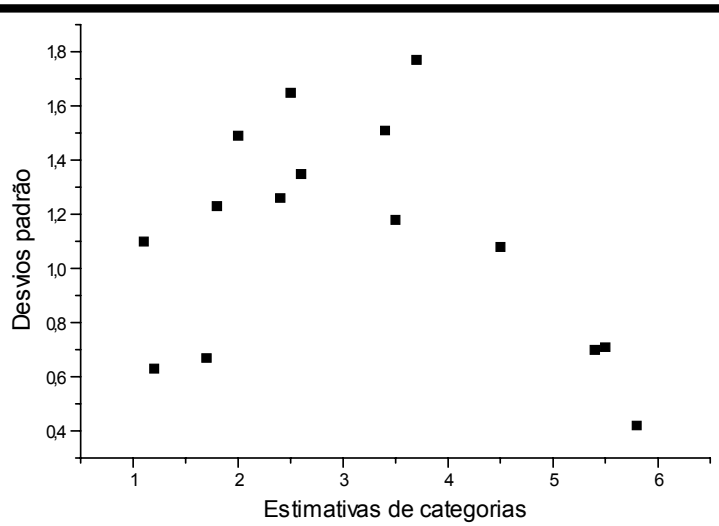

Figura 8 - Desvio padrão da média aritmética em função da média aritmética das estimativas de categorias da gravidade de quadros clínicos resultantes de cirurgias. Dados da Amostra 2

Existem algumas diferenças fundamentais nos escalonamentos obtidos. Com o método de estimação de categorias podemos apenas obter a ordenação e a diferença entre os graus de gravidade. Baseado nesse método não podemos afirmar o quanto o grau de gravidade de um dado quadro clínico é maior ou menor em comparação ao grau de um outro. Com o método de estimação de magnitudes podemos obter a ordenação, a diferença e, também, as razões entre os graus de gravidade dos diferentes quadros clínicos. Por exemplo, dos dados mostrados na Tabela 1, podemos afirmar que o grau de gravidade do quadro clínico resultante de aneurismectomia de aorta $(E M=378,75)$ é aproximadamente quatro vezes maior que o grau de gravidade do quadro clínico resultante de aritenoidectomia $(\mathrm{EM}=89,37)$, ou aproximadamente quatorze vezes maior que o grau de gravidade do quadro clínico resultante de fechamento de cistostomia $(E M=26,64)$. Da mesma forma, dos dados mostrados na Tabela 2, podemos afirmar que o grau de gravidade do quadro clínico resultante de revascularização do miocárdio $(\mathrm{EM}=279,60)$ é aproximadamente sete vezes maior que o grau de gravidade do quadro clínico resultante de fechamento de cistostomia $(E M=38,42)$, ou aproximadamente três vezes maior que o grau de gravidade do quadro clínico resultante de antrectomia $(\mathrm{EM}=98,95)$.

Para ilustrar estas vantagens pode-se mencionar o estudo de SCHEPP (1991) que comparou o método de estimação de magnitudes com a escala de Likert nos esforços de mães em manipularem suas energias físicas e emocionais para lidarem com suas crianças hospitalizadas. Os resultados mostraram que as respostas de razão derivadas do método de estimação de magnitude foram substancialmente diferentes daquelas obtidas com a Escala de Likert quanto às intensidades dos estímulos, 
portanto, sugerindo que estimação de magnitudes é superior em detectar variações com níveis de estímulos elevados, uma vez que com este método a amplitude de respostas é ilimitada. Sennott-Miller, Murdaugh e
Hinshaw (1988) registraram que os resultados obtidos através de estimação de magnitude produzem freqüentemente altos coeficientes de fidedignidade (produto-momento) teste-reteste, isto é, ao redor de 0,91 .

\section{GRAVITY OF CLINICAL ILLNESSES RESULTANT OF SURGERIES: MAGNITUDE ESTIMATION AND CATEGORIES ESTIMATION}

The gravity of clinical illnesses resultant of surgeries was scaled by the psychophysics methods of magnitude and categories estimations by 20 physicians and 20 nurses. Results showed: 1- the continuum of gravity of clinical illnesses resultant of surgeries has quantitative or prosthetic characteristics and non-qualitative or metathetic characteristics. 2- the variability of the estimations of magnitudes is a linear function of the magnitude of the estimations according to Ekman's law.

KEY WORDS: gravity, psychophysics

\section{MEDICIÓN DE LA GRAVEDAD DE CUADROS CLINICOS RESULTANTES DE CIRUGIAS: ESTIMACIÓN DE MAGNITUDES VERSUS ESTIMACIÓN DE CATEGORÍAS}

La gravedad de los cuadros clínicos que resultan de cirugías, fue escalonada por los métodos psicofísicos de estimación de magnitudes y de categorías por 20 médicos y 20 enfermeros. Los resultados mostraron: 1) El continuo de gravedad de cuadros clínicos resultantes de cirugías tiene características cuantitativas o protéticas y no cualitativas o metatéticas;2) La variabilidad de las estimativas de magnitudes es una función linear de la magnitud de las estimativas, según la ley de Ekman.

TÉRMINOS CLAVES: gravedad, psicofisica

\section{REFERÊNCIAS BIBLIOGRÁFICAS}

01. FALEIROS SOUSA, F.A.E.; DA SILVA, J.A. Uso e aplicação da metodologia psicofísica na pesquisa em enfermagem. Rev. Latino-am. Enfermagem, v. 4, p. 147-178, 1996 a.

02. FALEIROS SOUSA, F.A.E.; Da SILVA, J.A. Psicofísica do prestígio social: comparação entre estimação de magnitudes e de comparação aos pares. Arq. Bras. Psicol., v. 48, p. 69-79, 1996 b.

03. FALEIROS SOUSA, F.A.E.; KAMIZAKI, R.; SANT'ANA, R.P.M.; GIUNTINI, P.B. Mensuração da gravidade de quadros clínicos resultantes de cirurgias. Medicina, v. 31, n. 4, p. 616-625, 1998.

04. GESCHEIDER, G.A. Psychophysic: the fundamentals. Mahwah, New Jersey: LEA, 1997.

05. MEEK, P.M.; SENNOTT-MILLER, L.; FERKETICH, S.L. Scaling stimuli with magnitude estimation. Res. Nurs.Health, v. 15, p. 77-81, 1992.

06. PEREIRA, L.V.; FALEIROS SOUSA, F.A.E. Mensuração e avaliação da dor pós-operatória: uma breve revisão. Rev. Latinoam.Enfermagem, v. 6, p. 77-84, 1998a.

07. PEREIRA, L.V.; FALEIROS SOUSA, F.A.E. Estimação em categorias dos descritores da dor pós-operatória. Rev. Latino-am.Enfermagem, v. 6, p. $41-48,1998$ b.
08. SCHEPP, K. G. Factors influencing the coping effort of mothers of hospitalized children. Nurs.Res., v. 40, p. 42-46, 1991.

09. SENNOTT-MILLER, L.; MURDAUGH, C.; HINSHAW, A.S. Magnitude estimation: Issues and practical application. West. J. Nurs.Res., v. 10, p. 414-424, 1988.

10. STEVENS, S.S. A metric for the social consensus. Science, v. 151, p. 530-541, 1966.

11. STEVENS, S.S. (Ed.). Psychophysics: introduction to its perceptual, neural and social prospects. New York: Wiley, 1975.

12. VOLICER, B.J.; BOHANNON, M.W. A hospital stress rating scale. Nurs. Res., v. 24, p. 352-364, 1975.

13. WYLER, A.R.; MASUDA, M.; HOLMES, T.H. Seriousness of illness rating scale. J. Psychosom. Res., v. 11, p. 363-374, 1968.

14. WYLER, A.R.; MASUDA, M.; HOLMES, T.H. The seriousness of illness rating scale: Reproducibility. J. Psychosom. Res., v. 14, p. 59-64, 1970.

15.WYLER, A.R.; MASUDA, M.; HOLMES, T.H. Magnitude of life events and seriousness of illness. Psychosom. Med., v. 33, p. 115-122, 1971. 